\title{
Abelian quotients of the string link monoid
}

\author{
JEAN-BAPTISTE MEILHAN \\ AKIRA YASUHARA
}

\begin{abstract}
The set $\mathcal{S L}(n)$ of $n$-string links has a monoid structure, given by the stacking product. When considered up to concordance, $\mathcal{S L}(n)$ becomes a group, which is known to be abelian only if $n=1$. In this paper, we consider two families of equivalence relations which endow $\mathcal{S L}(n)$ with a group structure, namely the $C_{k}$-equivalence introduced by Habiro in connection with finite-type invariants theory, and the $C_{k}$-concordance, which is generated by $C_{k}$-equivalence and concordance. We investigate under which condition these groups are abelian, and give applications to finite-type invariants.
\end{abstract}

57M25, 57M27; 20F38

\section{Introduction}

For a positive integer $n$, let $D^{2}$ be the standard two-dimensional disk equipped with $n$ marked points $x_{1}, \ldots, x_{n}$ in its interior. Let $I$ denote the unit interval. An $n$-string link is a proper embedding

$$
L: \bigsqcup_{i=1}^{n} I_{i} \rightarrow D^{2} \times I
$$

of the disjoint union $\bigsqcup_{i=1}^{n} I_{i}$ of $n$ copies of $I$ in $D^{2} \times I$, such that for each $i$ the image $L_{i}$ of $I_{i}$ runs from $\left(x_{i}, 0\right)$ to $\left(x_{i}, 1\right)$. Abusing notation, we will also denote by $L \subset D^{2} \times I$ the image of the map $L$, and $L_{i}$ is called the $i^{\text {th }}$ string of $L$. Note that each string of an $n$-string link is equipped with an (upward) orientation induced by the natural orientation of $I$. The string link $\bigsqcup_{i=1}^{n}\left(\left\{x_{i}\right\} \times I\right)$ is called the trivial $n-$ string link and is denoted by $\mathbf{1}_{n}$, or simply by $\mathbf{1}$.

The set $\mathcal{S L}(n)$ of isotopy classes of $n$-string links fixing the endpoints has a monoid structure, with composition given by the stacking product and with the trivial $n$-string link $\mathbf{1}_{n}$ as unit element. There is a surjective map from $\mathcal{S L}(n)$ to the set of isotopy classes of $n$-component links, which sends an $n$-string link to its closure (in the usual sense). For $n=1$, this map is a monoid isomorphism.

It is well known that the monoid $\mathcal{S L}(n)$ is not a group. In fact, it is quite far from being a group: the group of invertible elements in $\mathcal{S L}(n)$ is actually the pure braid group 
on $n$ strands; see Habegger and Lin [11]. However, $\mathcal{S L}(n)$ becomes a group when considered up to concordance. Recall that two $n$-string links $L, L^{\prime}$ are concordant if there is an embedding

$$
f:\left(\bigsqcup_{i=1}^{n} I_{i}\right) \times I \longrightarrow\left(D^{2} \times I\right) \times I
$$

such that $f\left(\left(\bigsqcup_{i=1}^{n} I_{i}\right) \times\{0\}\right)=L$ and $f\left(\left(\bigsqcup_{i=1}^{n} I_{i}\right) \times\{1\}\right)=L^{\prime}$, and such that we have $f\left(\partial\left(\bigsqcup_{i=1}^{n} I_{i}\right) \times I\right)=(\partial L) \times I$. The inverse of a string link up to concordance is simply its horizontal mirror image with reversed orientation. Le Dimet showed that the group of concordance classes of $n$-string links is not abelian for $n \geq 3$ in [14]. ${ }^{1}$ The fact that this result also hold for $n=2$ seem to have been first observed by De Campos [3], as a consequence of a result of Miyazaki for the theta-curve cobordism group [19].

Now, other equivalence relations are known to endow $\mathcal{S L}(n)$ with a group structure: for each $k \geq 1$, the set $\mathcal{S L}(n) / C_{k}$ of $C_{k}$-equivalence classes of $n$-string links is a group; see Habiro [13]. Here, the $C_{k}$-equivalence is an equivalence relation on links generated by $C_{k}$-moves and ambient isotopies, defined in connection with the theory of finite-type invariants. A $C_{1}$-move is just a crossing change, and for any integer $k \geq 2$, a $C_{k}$-move is a local move on links as illustrated in Figure 1.1. These local moves can also be defined in terms of 'insertion' of an element of the $k^{\text {th }}$ lower central subgroup of some pure braid group (see Stanford [23]), or alternatively by using the theory of claspers (see Section 2).

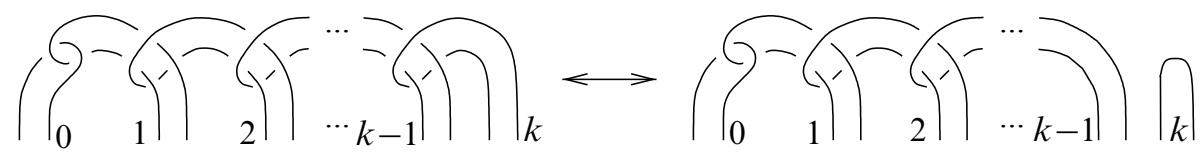

Figure 1.1: A $C_{k}$-move involves $k+1$ strands of a link, labelled here by integers between 0 and $k$.

Habiro proved in [13] that the quotient $\mathcal{S L}(n) / C_{k}$ is a finitely generated nilpotent group, for any $n$ and $k$. In this paper, we investigate under which condition this group is abelian. This is immediate for $k=1$ since $\mathcal{S L}(n) / C_{1}$ is the trivial group. It is also well known that, for all $n \geq 1$, the group $\mathcal{S L}(n) / C_{2}$ is abelian. This essentially follows from the fact, due to Murakami and Nakanishi [20], that $C_{2}$-equivalence classes of (string) links are classified by the linking number. Another rather easy fact is the following, which uses Milnor's triple linking number (see Section 4.3).

1 The literature sometimes erroneously refers to [14] for the analogous fact for $n=2$, but, as Le Dimet writes in [14, 4.5 Conclusion 2], his arguments did not allow him to conclude in the case of 2-string links. 
Proposition 1.1 The group $\mathcal{S L}(n) / C_{k}$ of $C_{k}$-equivalence classes of $n$-string links is not abelian for any $n \geq 3$ and any $k \geq 3$.

Hence our study is reduced to the case of 2-string links. In [25], the second author showed that $\mathcal{S L}(2) / C_{k}$ is not abelian for $k \geq 12$. Here we improve this result and give an almost complete answer for this 'abelian problem.'

Theorem 1.2 (1) The group $\mathcal{S L}(2) / C_{k}$ of $C_{k}$-equivalence classes of 2 -string links is abelian for $k<7$.

(2) The group $\mathcal{S L}(2) / C_{k}$ is not abelian for $k>7$.

Although the case $k=7$ remains open so far, we show the following.

Theorem 1.3 The group $\mathcal{S L}(2) / C_{7}$ is abelian if it has no 2 -torsion.

Remark 1.4 Bar-Natan's computations [2] show that 7 is the smallest degree of a finite-type invariant that can detect the orientation of 2-string links. The existence of such an invariant was shown by Duzhin and Karev [8], and this result is used in Section 3.2 to prove Theorem 1.2(2). Likewise, it would be very interesting to determine whether there exists a $\mathbb{Z}_{2}$-valued finite-type invariant of degree 6 that can detect the orientation of 2 -string links.

The 'abelian problem' addressed above is deeply related to one of the main results in the theory of finite-type invariants, due to Habiro [13] and Goussarov [10] independently, which gives a topological characterization of the information contained by finite-type invariants of knots.

Theorem 1.5 [10; 13] Two knots cannot be distinguished by any finite-type invariant of order less than $k$ if and only if they are $C_{k}$-equivalent.

It was indeed conjectured by both Goussarov and Habiro that, although it fails to hold for links, Theorem 1.5 may generalize to string links. One of the key ingredients of Habiro's proof of Theorem 1.5, based on the theory of claspers, is the fact that the set of $C_{k}$-equivalence classes of knots forms an abelian group for all $k \geq 1$. As a matter of fact, his techniques apply to the string link case, and the fact that the group $\mathcal{S L}(n) / C_{k}$ is abelian implies that the conjecture holds at the corresponding degree (see the Appendix for further explanation). Hence Theorem 1.2(1) gives us the following.

Corollary 1.6 For any integer $k \leq 6$, two $2-$ string links cannot be distinguished by any finite-type invariant of order less than $k$ if and only if they are $C_{k}$-equivalent. 
Remark 1.7 The Goussarov-Habiro Conjecture for string links was shown to be true at low degree by various authors. It is easy to check for $k=2$, using the linking number [20], and was proved for $k=3$ by the first author in [16]. Massuyeau gave a proof for $k=4$, using algebraic arguments [15]. In [17], the authors classified string links up to $C_{k}$-equivalence for $k \leq 5$, by explicitly giving a complete set of low degree finite-type invariants, and proved the Goussarov-Habiro conjecture for $k \leq 5$ as a byproduct.

Since we have two equivalence relations, concordance and $C_{k}$-equivalence, that provide group structures on the set of string links, it is natural to combine them to get a new group structure. We call this equivalence relation on string links generated by $C_{k}-$ moves and concordance the $C_{k}$-concordance [17]. The $C_{k}$-concordance is very closely related to Whitney tower concordance of order $k-1$ studied in [6] by Conant, Schneiderman and Teichner, ${ }^{2}$ and to finite-type concordance invariants.

In Section 4, we investigate whether the group $\mathcal{S L}(n) /\left(C_{k}+c\right)$ of $C_{k}$-concordance classes of $n$-string links is abelian. In the knot case, it is known that $\mathcal{S L}(1) /\left(C_{k}+c\right)$ is trivial for $k=1$ or 2 , and that $\mathcal{S L}(1) /\left(C_{k}+c\right)$ is isomorphic to $\mathbb{Z} / 2 \mathbb{Z}$ for $k \geq 3$; see $\mathrm{Ng}$ [21] and the authors [17]. We show the following.

Theorem 1.8 (1) The group $\mathcal{S L}(2) /\left(C_{k}+c\right)$ is abelian if and only if $k \leq 8$.

(2) For $n \geq 3$, the group $\mathcal{S L}(n) /\left(C_{k}+c\right)$ is abelian if and only if $k \leq 2$.

The proof of (1) is given in Sections 4.4 and 4.5. In Section 4.3, we prove the 'only if' part in (2). The 'if' part is actually easy to see. Indeed, as mentioned above, $\mathcal{S L}(n) / C_{1}$ is a trivial group and $\mathcal{S L}(n) / C_{2}$ is an abelian group classified by the linking number. Since the latter is a $C_{2}$-concordance invariant, we have that $\mathcal{S L}(n) / C_{2}$ and $\mathcal{S L}(n) /\left(C_{2}+c\right)$ are isomorphic.

Since two $C_{k}$-concordant string links share all finite-type concordance invariants of degree less than $k$, and it is natural to ask, parallel to the Goussarov-Habiro conjecture, whether the converse is also true. This question was raised by the authors in [17]. As in the case of the $C_{k}$-equivalence, Habiro's arguments apply if $\mathcal{S L}(n) /\left(C_{k}+c\right)$ is abelian. Hence we have the following corollary.

Corollary 1.9 For any integer $k \leq 8$, two 2-string links cannot be distinguished by any finite-type concordance invariant of order less than $k$ if and only if they are $C_{k}$-concordant.

2 These two equivalence relations are actually equivalent, as announced in [6]. 
Acknowledgements The authors are grateful to Tetsuji Kuboyama, Hiroshi Sakamoto and Yoshimasa Takabatake for writing and improving greatly the computer program for Milnor invariants used in Section 4.5. They also thank Kazuo Habiro for discussions that led to Lemma 2.9, and the referee for several useful remarks which helped improve the paper significantly. The first author is supported by the French ANR research projects "VasKho" ANR-11-JS01-00201 and "ModGroup" ANR-11-BS01-02001. The second author is supported by a JSPS Grant-in-Aid for Scientific Research (C) (\#23540074).

\section{Claspers}

We recall here the main definitions and properties of the theory of claspers, which is one of the main tools of this paper. For convenience, we restrict ourselves to the case of string links. For a general definition of claspers, we refer the reader to [13].

\subsection{A brief review of clasper theory}

Let $L$ be a string link. An embedded surface $g$ is called a graph clasper for $L$ if it satisfies the following three conditions:

(1) $g$ is decomposed into disks and bands, called edges, each of which connects two distinct disks.

(2) The disks have either 1 or 3 incident edges, and are called leaves or nodes respectively.

(3) $g$ intersects $L$ transversely, and the intersections are contained in the union of the interiors of the leaves.

In particular, if a graph clasper is a simply connected, we call it a tree clasper. A graph clasper for a string link $L$ is simple if each of its leaves intersects $L$ at one point.

The degree of a connected graph clasper $g$ is defined as half the number of nodes and leaves. We call a degree $k$ connected graph clasper a $C_{k}$-graph. A tree clasper of degree $k$ is called a $C_{k}$-tree.

Convention 2.1 Throughout this paper, we make use of the drawing convention for claspers of [13, Figure 7], except for the following: a $\oplus$ (resp. $\ominus$ ) on an edge represents a positive (resp. negative) half-twist. (This replaces the convention of a circled $S$ (resp. $S^{-1}$ ) used in [13].) 
Given a graph clasper $g$ for a string link $L$, there is a procedure to construct, in a regular neighborhood of $g$, a framed link $\gamma(g)$. There is thus a notion of surgery along $g$, which is defined as surgery along $\gamma(g)$. There exists a canonical diffeomorphism between $D^{2} \times I$ and the manifold $\left(D^{2} \times I\right)_{\gamma(g)}$ fixing the boundary, and surgery along the $C_{k}$-graph $g$ can thus be regarded as an operation on $L$ in the (fixed) ambient space $D^{2} \times I$. We say that the resulting string link $L_{g}$ in $D^{2} \times I$ is obtained from $L$ by surgery along $g$. In particular, surgery along a simple $C_{k}$-tree is a local move as illustrated in Figure 2.1.

Throughout this paper, we will often define string links in terms of claspers for the trivial string link, implicitly referring to the result of surgery along this clasper.
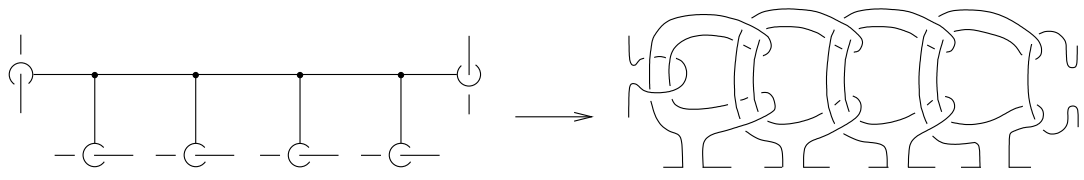

Figure 2.1: Surgery along a simple $C_{5}$-tree

The $C_{k}$-equivalence (as defined in the introduction) coincides with the equivalence relation on string links generated by surgeries along $C_{k}$-graphs and isotopies. In particular, it is known that two links are $C_{k}$-equivalent if and only if they are related by surgery along simple $C_{k}$-trees [13, Theorem 3.17].

This family of equivalence relations becomes finer as the degree increases, that is, the $C_{k}$-equivalence implies the $C_{m}$-equivalence if $k>m$.

A string link is called $C_{k}$-trivial if it is $C_{k}$-equivalent to the trivial string link.

\subsection{Standard calculus of claspers}

In this subsection, we summarize several properties of claspers. Although similar statements hold in a more general context, it will be convenient to state these results for the trivial string link 1. Proofs are omitted, since they involve the same techniques as in [13, Section 4], where similar statements appear.

Lemma 2.2 Let $t_{1} \cup t_{2}$ be a disjoint union of a $C_{k_{1}}$-graph and a $C_{k_{2}}$-graph for 1 . Let $t_{1}^{\prime} \cup t_{2}^{\prime}$ be obtained from $t_{1} \cup t_{2}$ by sliding a leaf of $t_{1}$ across a leaf of $t_{2}$; see Figure 2.2. Then

$$
\mathbf{1}_{t_{1} \cup t_{2}} \stackrel{C_{k_{1}+k_{2}+1}^{\sim}}{\sim} \mathbf{1}_{t_{1}^{\prime} \cup t_{2}^{\prime}} \cdot \mathbf{1}_{t},
$$

where $t$ is a $C_{k_{1}+k_{2}}$-graph obtained from $t_{1} \cup t_{2}$ by inserting a vertex $v$ in the edge $e$ of $t$ and connecting $v$ to the edge incident to $f$ as shown in Figure 2.2. 


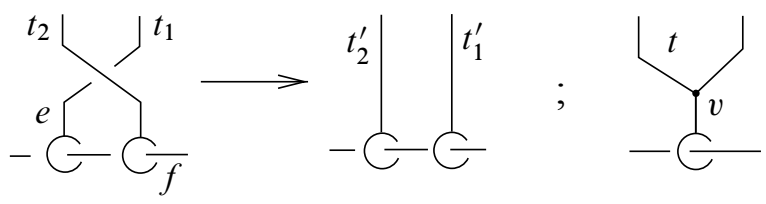

Figure 2.2: Sliding a leaf

Lemma 2.3 Let $t_{1} \cup t_{2}$ be a disjoint union of a $C_{k_{1}}$-graph and a $C_{k_{2}}$-graph for 1 . Let $t_{1}^{\prime} \cup t_{2}^{\prime}$ be obtained from $t_{1} \cup t_{2}$ by changing a crossing of an edge of $t_{1}$ with an edge of $t_{2}$. Then

$$
\mathbf{1}_{t_{1} \cup t_{2}} \stackrel{C_{k_{1}+k_{2}}+2}{\sim} \mathbf{1}_{t_{1}^{\prime} \cup t_{2}^{\prime}} \cdot \mathbf{1}_{t},
$$

where $t$ is a $C_{k_{1}+k_{2}+1}$-graph obtained by inserting a vertex in both edges involved in this crossing change, and connecting them by an edge.

Remark 2.4 By combining the two previous lemmas, we have the following. Let $L_{1}$ (resp. $L_{2}$ ) be a $C_{k_{1}}$-trivial (resp. $C_{k_{2}}$-trivial) $n$-string link, for some $n \geq 1$. Then $L_{1} \cdot L_{2}$ is $C_{k_{1}+k_{2}}$-equivalent to $L_{2} \cdot L_{1}$. This fact was already noted by Habiro in [13, Proposition 5.8].

Lemma 2.5 Let $g$ be a $C_{k}$-graph for 1 . Let $f_{1}$ and $f_{2}$ be two disks obtained by splitting a leaf $f$ of $g$ along an arc $\alpha$ as shown in Figure 2.3.
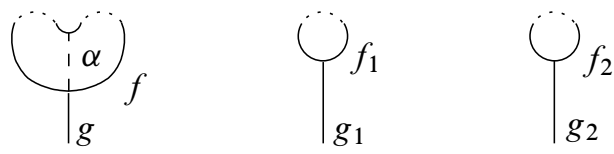

Figure 2.3: The 3 claspers are identical outside a small ball, where they are as depicted.

Then,

$$
\text { (1) } \stackrel{C_{k+1}}{\sim}(\mathbf{1})_{g_{1}} \cdot(\mathbf{1})_{g_{2}}
$$

where $g_{i}$ denotes the $C_{k}$-graph for 1 obtained from $g$ by replacing $f$ by $f_{i}(i=1,2)$; see Figure 2.3.

Lemma 2.6 Let $t$ be a $C_{k}$-graph for $\mathbf{1}$, and let $t^{\prime}$ be a $C_{k}$-graph obtained from $t$ by adding a half-twist on an edge. Then

$$
\mathbf{1}_{t} \cdot \mathbf{1}_{t^{\prime}} \stackrel{C_{k+1}}{\sim} \mathbf{1}
$$


Claspers also satisfy relations analogous to the AS, IHX and STU relations for Jacobi diagrams; see Bar-Natan [1].

Lemma 2.7 (AS) Let $t$ and $t^{\prime}$ be two $C_{k}$-graphs for 1 which differ only in a small ball as depicted in Figure 2.4.
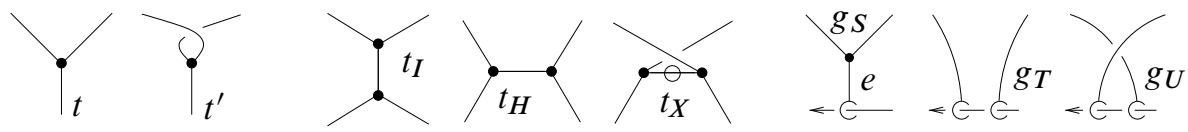

Figure 2.4: The AS, IHX and STU relations

Then

$$
\mathbf{1}_{t} \cdot \mathbf{1}_{t^{\prime}} \stackrel{C_{k+1}}{\sim} \mathbf{1} .
$$

(IHX) Let $t_{I}, t_{H}$ and $t_{X}$ be three $C_{k}$-graphs for 1 which differ only in a small ball as depicted in Figure 2.4. Then

$$
\mathbf{1}_{t_{I}} \stackrel{C_{k+1}}{\sim} \mathbf{1}_{t_{H}} \cdot \mathbf{1}_{t_{X}} .
$$

(STU) Let $g_{S}, g_{T}$ and $g_{U}$ be three $C_{k}$-graphs for 1 which differ only in a small ball as depicted in Figure 2.4. Then

$$
\mathbf{1}_{g_{S}} \cdot \mathbf{1}_{g_{T}} \stackrel{C_{k+1}}{\sim} \mathbf{1}_{g_{U}} .
$$

In the rest of the paper, we will simply refer to Lemma 2.7 as the AS, IHX and STU relations.

By the IHX and STU relations, one can easily check the following.

Lemma 2.8 Let $t$ be a $C_{k}$-graph for 1 .

(1) Suppose that there exists a 3-ball which intersects $t$ as on the left-hand side of Figure 2.5. Then

$$
\mathbf{1}_{t} \stackrel{C_{k+1}}{\sim} \mathbf{1}_{c},
$$

where $c$ is a $C_{k}$-graph as shown in the figure.

(2) Suppose that there exists a 3-ball which intersects $t$ as on the right-hand side of Figure 2.5. Then

$$
\mathbf{1}_{t} \stackrel{C_{k+1}}{\sim} \mathbf{1}_{g},
$$

where $g$ is a $C_{k}$-graph as shown in the figure. 

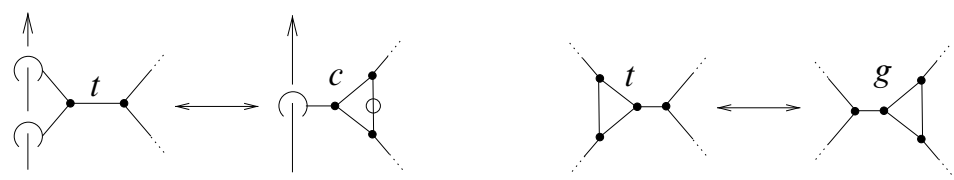

Figure 2.5

\subsection{A commutativity lemma}

We conclude this section with a purely algebraic lemma. Although seemingly very technical, it turns out to be a rather natural tool in the proofs of our main results.

Lemma 2.9 Let $L$ be an $n$-string link satisfying the following three conditions, for some integers $p, l, m$ and $k(p<k, l<m<k)$ :

- $L^{2}$ is $C_{p}$-equivalent to a central element in $\mathcal{S L}(n) / C_{k}$.

- $L$ commutes with any $C_{l}$-trivial string link in $\mathcal{S L}(n) / C_{m}$.

- $L$ commutes with any $C_{m}$-trivial string link in $\mathcal{S L}(n) / C_{k}$.

Suppose, moreover, that $\mathcal{S L}(n) / C_{k}$ has no 2-torsion, and that $C_{l}$-trivial and $C_{p}-$ trivial string links all commute in $\mathcal{S L}(n) / C_{k}$. Then $L$ commutes with any $C_{l}$-trivial string link in $\mathcal{S L}(n) / C_{k}$.

Remark 2.10 Observe that a string link commutes with any $C_{l}$-trivial string link in $\mathcal{S L}(n) / C_{k}(l<k)$ if and only if it commutes with $\mathbf{1}_{t}$ for any $C_{s}$-tree $t$ with $l \leq s \leq k-1$. Indeed, let $T$ and $T^{\prime}$ be two $C_{l}$-equivalent string links. Since $T$ is $C_{1}$-trivial, it is not hard to see, using Lemmas 2.2 and 2.3, that $T^{\prime}$ is $C_{k}$-equivalent to a product $T \cdot T_{l} \cdot T_{l+1} \cdots T_{k-1}$, where $T_{i}$ is a product of string links, each obtained from 1 by surgery along a single $C_{i}$-tree $(l \leq i \leq k-1)$. In particular, each $T_{i}$ is a $C_{i}$-trivial string link, and $T_{l} \cdot T_{l+1} \cdots T_{k-1}$ is thus a $C_{l}$-trivial string link.

Proof As mentioned above, this is a purely algebraic result, which relies on the following.

Fact Let $A, B$ and $C$ be subgroups of some group $G$. Let $x, z \in G$, where $z$ is central. Suppose that

$$
x^{2} z^{-1} \in C, \quad[x, A] \subset B, \quad[x, B]=\{1\}, \quad[A, C]=\{1\} .
$$

If $B$ has no 2-torsion, then we have that $[x, A]=\{1\}$. (Here, $[g, h]$ denotes the commutator $g h g^{-1} h^{-1}$ of $g$ and $h$.) 
This is easily shown as follows. Set $c=x^{2} z^{-1} \in C$. Then, for any element $a$ of $A$, the above assumptions imply that

$$
[x, a]^{2}=x[x, a] x^{-1}[x, a]=\left[x^{2}, a\right]=[c z, a]=[c, a]=1,
$$

which implies that $[x, a]=1$ as desired, since $B$ has no 2 -torsion.

Lemma 2.9 is merely an application of this fact in the case where $G=\mathcal{S L}(n) / C_{k}$, $A=\mathcal{S L}_{l}(n) / C_{k}, B=\mathcal{S L}_{m}(n) / C_{k}$ and $C=\mathcal{S L}_{p}(n) / C_{k}$, where $\mathcal{S L}_{i}(n) / C_{k}$ denotes the subgroup of $\mathcal{S L}(n) / C_{k}$ of the $C_{k}$-equivalence classes of $C_{i}$-trivial string links.

Remark 2.11 It follows from the above proof that Lemma 2.9 still holds if we replace, in the statement, the condition that $\mathcal{S L}(n) / C_{k}$ has no 2-torsion with the condition that $\mathcal{S L}_{m}(n) / C_{k}$ has no 2-torsion.

\section{The group of $C_{k}$-equivalence classes of 2-string links}

In this section, we prove Theorems 1.2 and 1.3.

\subsection{Abelian cases: $k \leq 6$}

Since the $C_{k}$-equivalence implies the $C_{m}$-equivalence if $k>m$, it is sufficient to show that $\mathcal{S L}(2) / C_{6}$ is abelian. This is done in Proposition 3.3, by first providing a set of generators for this group and then by showing that any two of these generators commute in $\mathcal{S L}(2) / C_{6}$. (Recall that it was shown by Habiro that $\mathcal{S L}(n) / C_{k}$ is a finitely generated group for any $n$ and $k$ [13].)

Let the 2-string links $I, Y, Y^{\prime}, H, X, D, S_{\alpha}^{1}$ and $S_{\alpha}^{2}\left(\alpha \in S_{3}\right)$ obtained from $\mathbf{1}_{2}$ by surgery along the tree clasper $i, y, y^{\prime}, h, x, d, s_{\alpha}^{1}$ and $s_{\alpha}^{2}$, represented in Figure 3.1, respectively.
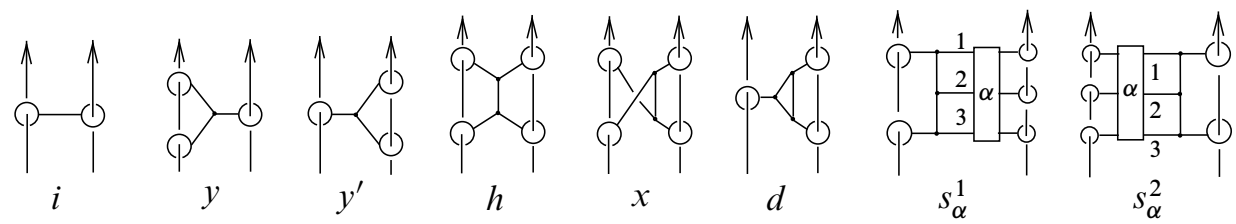

Figure 3.1

Let $\mathcal{S L}_{i}$ (2) denote the set of $C_{i}$-trivial 2-string links ( $i \geq 1$ ). It is shown in [17, Sections 4.1-4.3.2] that, for $i \in\{1,2,3,4\}$, the group $\mathcal{S L}_{i}(2) / C_{i+1}$ has generating 
set $\mathcal{H}_{i}=\mathcal{H}_{i}^{m} \cup \mathcal{H}_{i}^{l}$, where ${ }^{3}$

$$
\mathcal{H}_{1}^{m}=\{I\}, \quad \mathcal{H}_{2}^{m}=\{Y\}, \quad \mathcal{H}_{3}^{m}=\{H, D\}, \quad \mathcal{H}_{4}^{m}=\left\{\sigma_{\mathrm{id}}^{1}, \sigma_{(12)}^{1}, \sigma_{(123)}^{1}, \sigma_{(13)}^{1}, \sigma_{\mathrm{id}}^{2}\right\},
$$

and where $\mathcal{H}_{i}^{l}$ is a set of local generators. Here, we say that an element of $\mathcal{S L}(2)$ is local if there exists a 3-ball whose boundary intersects it at only two points, such that an homotopy of this ball to a point produces the trivial 2-string link. (In other words, local elements consists of a local knot on one strand.) Clearly local elements are central in $\mathcal{S} \mathcal{L}(2)$.

Let us also fix a generating set $\mathcal{H}_{5}$ for $\mathcal{S L}_{5}(2) / C_{6}$. We call elements of $\mathcal{H}_{i}(i \leq 5)$ generators of degree $i$.

Before stating Proposition 3.3, we make a few simple, yet useful observations. First, notice the following.

Lemma 3.1 The string link $I$ is central in $\mathcal{S L}(2)$.

Proof This is shown in Figure 3.2. Let $L \in \mathcal{S L}(2)$. Then $I \cdot L$ is as represented on the
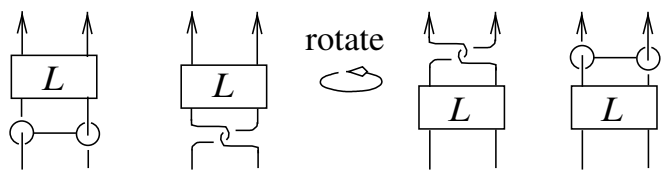

Figure 3.2

left-hand side of the figure. Rotating the two strings by 360 degrees about the vertical axis (fixing the endpoints) yields an isotopic string link, which is precisely $L \cdot I$.

Next, we have the following.

Fact 3.2 The string links $Y$ and $Y^{\prime}$ are ambient isotopic.

This is the string link version of the usual symmetry property of the Whitehead link, as illustrated in Figure 3.3 below.

In the following, however, we will use the term 'symmetric' for another property. Given an $n$-string link $L$, we denote by $\bar{L}$ its image under orientation-reversal of all its strands. The string link $L$ is called symmetric if it is isotopic to $\bar{L}$. For example, the

${ }^{3}$ In [17], the generating set $\mathcal{H}_{3}^{m}=\{H, X\}$ is used instead. Here, it will be convenient to use the element $D$ rather than $X$ as generator. This is possible since, by the STU relation, we have $D \cdot H \stackrel{C_{4}}{\sim}$. 


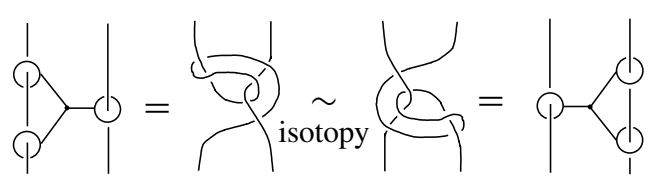

Figure 3.3: Symmetry property of the Whitehead string link

string links $I, Y, Y^{\prime}, H$ and $D$ are all symmetric. Notice that this is nicely reflected in the symmetry of the clasper defining these string links.

We can now prove the first part of Theorem 1.2.

Proposition 3.3 The group $\mathcal{S L}(2) / C_{6}$ is abelian.

Proof We use the generating sets $\mathcal{H}_{i}$ for $\mathcal{S L}_{i}(2) / C_{i+1}$ specified above $(1 \leq i \leq 5)$. In order to prove Proposition 3.3, it suffices to show that lifts in $\mathcal{S L}(2) / C_{6}$ of any two elements of $\bigcup_{i=1}^{5} \mathcal{H}_{i}$ commute, since $\bigcup_{i=1}^{5} \mathcal{H}_{i}$ forms a generating set for this group.

By Remark 2.4, two generators of degree $k$ and $k^{\prime}$ commute in $\mathcal{S L}(2) / C_{6}$ if $k+k^{\prime} \geq 6$. Hence all generators of degree 5 are central in $\mathcal{S L}(2) / C_{6}$. Remark 2.4 also implies that $H$ and $D$ commute with each other. Moreover, we may safely ignore local generators, since these are central elements in $\mathcal{S L}(2)$, as well as $I$ (by Lemma 3.1).

So we only need to check that the generator of degree 2 , namely $Y$, commutes with both $H$, and $D$.

Let us first show that $Y$ and $H$ commute in $\mathcal{S L}(2) / C_{6}$. The proof is given in Figures 3.4 and 3.5 as follows. Consider the product $H \cdot Y$. By Lemmas 2.2 and 2.6, we have that $H \cdot Y \stackrel{C_{6}}{\sim} G \cdot K$, where $G$ and $K$ are shown in Figure 3.4. Observe that $G$ 'locally'
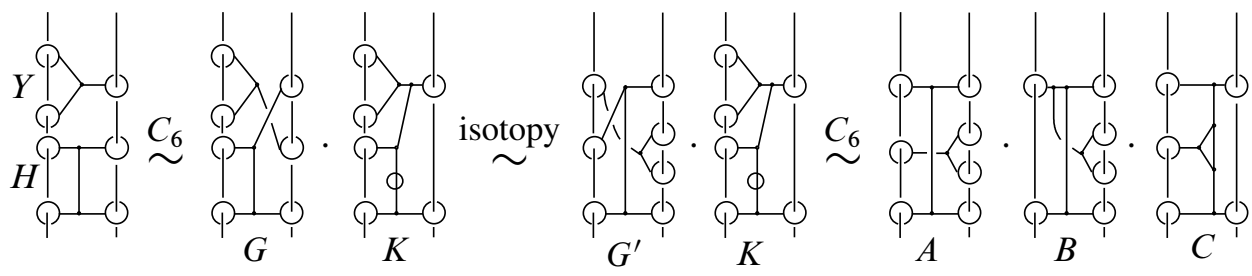

Figure 3.4

contains a copy of $Y$, so that we can use Fact 3.2 to show that $G$ is isotopic to the string link $G^{\prime}$ represented in Figure 3.4. Now, by a second application of Lemma 2.2, $G^{\prime}$ is $C_{6}$-equivalent to the product of the two string links $A$ and $B$ represented on the 
right-hand side of the figure. Also, Lemmas 2.6 and 2.8 imply that $K \stackrel{C_{6}}{\sim} C$, where $C$ is also given in Figure 3.4.

Let us now focus on the string link $B$. By the IHX relation and Lemma 2.6, we have $B \sim B^{C_{6}} \cdot B^{\prime \prime}$, as illustrated in Figure 3.5. Applying Lemmas 2.8 and 2.6 to the string
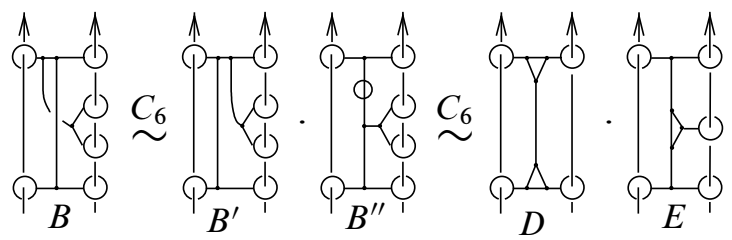

Figure 3.5

link $B^{\prime}$ and $B^{\prime \prime}$, we deduce that $B \stackrel{C_{6}}{\sim} D \cdot E$, where $D$ and $E$ are represented on the right-hand side of Figure 3.5.

Hence we have shown that $H \cdot Y \stackrel{C_{6}}{\sim} A \cdot C \cdot D \cdot E$. Note that, by Remark 2.4, we have that $A, C, D$ and $E$ commute with each other in $\mathcal{S L}(2) / C_{6}$. Observe that $A \sim C_{6} \bar{A}$ by Lemma 2.3, and that the three string links $C, D$ and $E$ are symmetric. We thus have

$$
H \cdot Y \stackrel{C_{6}}{\sim} \overline{H \cdot Y}=Y \cdot H,
$$

where the equality follows from the fact that $Y$ and $H$ are also symmetric.

Similarly, we now show that $Y$ commutes with $D$ as follows. Starting with the product $D \cdot Y$ and applying Lemmas 2.2 and 2.6 twice as shown in Figure 3.6, we obtain that $D \cdot Y \sim C_{6} M \cdot N \cdot O$, where $M, N$ and $O$ are represented on the right-hand side of the figure. The STU relation shows that $N \stackrel{C_{6}}{\sim} P \cdot Q$, where $P$ and $Q$ are represented in
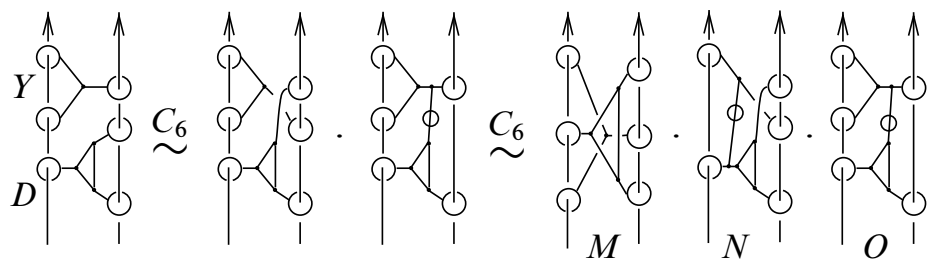

Figure 3.6

Figure 3.7. Now, by Lemmas 2.6 and 2.8 we see that $O$ and $P$ are both $C_{6}$-equivalent to the string link $R$ shown in Figure 3.7. Summarizing, we have shown that

$$
D \cdot Y \stackrel{C_{6}}{\sim} M \cdot R^{2} \cdot Q
$$




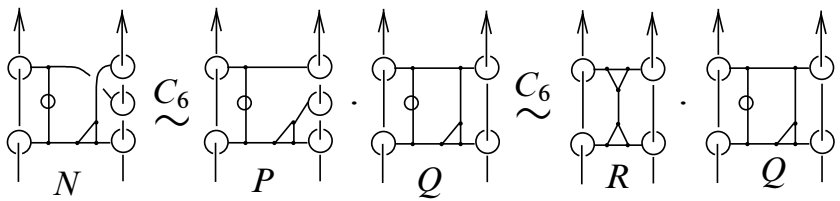

Figure 3.7

Now, we have that $M \stackrel{C_{6}}{\sim} \bar{M}$ by Lemma 2.3, and also $Q \stackrel{C_{6}}{\sim} \bar{Q}$ by Lemma $2.8(2)$. Hence we obtain that $D \cdot Y \sim C_{6} \overline{D \cdot Y}=Y \cdot D$, where the equality follows from the fact that $Y$ and $D$ are both symmetric.

\subsection{Nonabelian case: $k \geq 8$}

Denote by $\mathcal{A}(2)$ the $\mathbb{Q}$-vector space of Jacobi diagrams on two strands, modulo the Framing Independance (FI) and STU relation, and denote by $\mathcal{A}_{k}(2)$ the subspace generated by degree $k$ elements (see eg [1] for the definitions). The stacking product . endows $\mathcal{A}(2)$ with an algebra structure. As is well known, $\mathcal{A}(2)$ coincides with the space of chord diagrams on two strands modulo the FI and 4T relations [1].

In [8], Duzhin and Karev showed that $\mathcal{A}(2) / \mathcal{A}_{8}(2)$, hence $\mathcal{A}(2)$, is noncommutative. More precisely, let $D_{H} \in \mathcal{A}_{3}(2)$ and $D_{S} \in \mathcal{A}_{4}$ (2) be the two Jacobi diagrams shown in Figure 3.8. Then we have that $D_{H} \cdot D_{S} \neq D_{S} \cdot D_{H}$ in $\mathcal{A}_{7}(2)$ [8, Proposition 1].

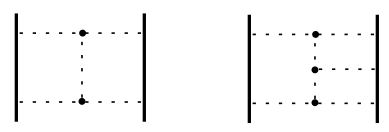

Figure 3.8: The Jacobi diagrams $D_{H} \in \mathcal{A}_{3}(2)$ and $D_{S} \in \mathcal{A}_{4}(2)$

We now show how this result implies that $\mathcal{S L}(2) / C_{8}$ is not abelian.

For $k \geq 0$, let $J_{k}(2)$ denote the subgroup of $\mathbb{Z} \mathcal{S L}(2)$ generated by singular 2-string links with $k$ double points, via the Vassiliev skein relation:

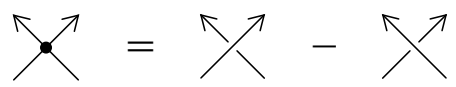

By definition, the difference of two string links is in $J_{k+1}(2)$ if and only if they cannot be distinguished by any finite-type invariant of degree less than or equal to $k$. There is a well-known isomorphism

$$
\xi_{k}: \mathcal{A}_{k}(2) \rightarrow\left(J_{k}(2) / J_{k+1}(2)\right) \otimes \mathbb{Q}
$$

which "maps chords to double points", with inverse given by the Kontsevich integral. 
Now recall from Section 3.1 that $H$ and $S_{\text {Id }}^{1}$ denote the 2-string links obtained from $\mathbf{1}_{2}$ by surgery along the tree clasper $h$ and $s_{\text {Id }}^{1}$ represented in Figure 3.1, respectively. (For simplicity, we will use here the simpler notation $S$ for the string link $S_{\text {Id }}^{1}$.) Then the image of $D_{H} \cdot D_{S}-D_{S} \cdot D_{H}$ by $\xi_{7}$ is (up to a sign) the difference $H \cdot S-S \cdot H$ (this can be checked using a standard argument on the good behavior of the Kontsevich integral on alternate sums defined by claspers; see eg Ohtsuki [22, Appendix E]). This shows that $H \cdot S$ and $S \cdot H$ can be distinguished by some degree 7 finite-type invariant. Since two $C_{k}$-equivalent (string) links cannot be distinguished by any finite-type invariant of degree less than $k$, we deduce that $H \cdot S$ and $S \cdot H$ are not $C_{8}$-equivalent.

\subsection{The case $k=7$}

In this section, we prove Theorem 1.3 , hence we suppose that $\mathcal{S L}(2) / C_{7}$ has no 2-torsion.

We proceed as in Section 3.1, using the generating sets $\mathcal{H}_{i}$ for $\mathcal{S L}_{i}(2) / C_{i+1}(i \leq 5)$. By Remark 2.4, elements of $\mathcal{S L}_{6}(2) / C_{7}$ are central in $\mathcal{S L}(2) / C_{7}$, so we only need to consider these generators of degree less than or equal to 5 . As above, we may also safely ignore local generators, as well as $I$. Actually, by Lemma 3.1 and Remark 2.4, we only have to check the following commutativity properties:

$(3,3)$ Generators of degree 3 commute with each other.

$(2,4)$ Generators of degree 4 commute with generators of degree 2.

$(2,3)$ Generators of degree 3 commute with generators of degree 2.

(Recall from Section 3.1 that by generator of degree $k$ we mean any element of $\mathcal{H}_{k}$.)

Case $(3,3)$ We have to show that $H$ and $D$ commute in $\mathcal{S L}(2) / C_{7}$. We will use the following result, whose proof is postponed to the end of this section.

Claim 3.4 The string link $D^{2}$ is $C_{4}$-equivalent to a central element in $\mathcal{S L}(2)$.

It follows from this fact and Remark 2.4 that $D$ fulfills the assumptions of Lemma 2.9 with $(p, l, m, k)=(4,3,6,7)$. Applying the lemma then proves that $D$ commutes with any $C_{3}$-trivial string link in $\mathcal{S L}(2) / C_{7}$, and in particular with $H$.

Case $(2,4)$ We have the following.

Claim 3.5 The string link $Y^{2}$ is $C_{3}$-equivalent to a central element in $\mathcal{S L}(2)$. 
(The proof of Claim 3.5 uses the exact same arguments as the proof of Claim 3.4, although it turns out to be actually significantly simpler.)

Claim 3.5 and Remark 2.4 ensure that $Y$ satisfies the assumptions of Lemma 2.9 with $(p, l, m, k)=(3,4,6,7)$. Applying the lemma then shows that $Y$ commutes with any generator of degree 4 in $\mathcal{S L}(2) / C_{7}$.

Case $(\mathbf{2}, \mathbf{3})$ Using Case $(\mathbf{3}, \mathbf{3})$ and Claim 3.5, we can now apply Lemma 2.9 to the string link $Y$ with $(p, l, m, k)=(3,3,5,7)$. Applying the lemma then shows that $Y$ commutes with any generator of degree 3 in $\mathcal{S L}(2) / C_{7}$.

We conclude this section with the proof of Claim 3.4.

Proof of Claim 3.4 We first notice that, as a consequence of Lemma 2.8, the string link $D$ has a symmetry property similar to that of the Whitehead string link $Y$ in $\mathcal{S L}(2) / C_{4}$ (see Figure 3.3):

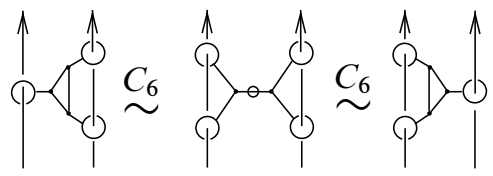

Figure 3.9: A Whitehead-type symmetry for the 2-string link $D$

Now consider the $C_{3}$-graph $g$ for $\mathbf{1}_{2}$ shown on the left-hand side of Figure 3.10. Clearly, the 2 -string link $G$ obtained by surgery along $g$ is central in $\mathcal{S L}(2)$. By applying repeatedly Lemma 2.5 , we have that $G$ is $C_{4}$-equivalent to a product $\prod_{i=1}^{8}\left(\mathbf{1}_{2}\right)_{g_{i}}$ of eight 2 -string links, each obtained from $\mathbf{1}_{2}$ by surgery along a simple $C_{4}$-graph, as shown in Figure 3.10. By several isotopies, Lemma 2.6 and the symmetry property of Figure 3.9 , we deduce that $G$ is $C_{4}$-equivalent to the product $C \cdot\left(D^{*}\right)^{2}$, where $C=\left(\mathbf{1}_{2}\right)_{g_{1}} \cdot\left(\mathbf{1}_{2}\right)_{g_{5}}$ is clearly central in $\mathcal{S L}(2)$ and where $D^{*}=\left(\mathbf{1}_{2}\right)_{d^{*}}$ (see Figure 3.10).

Using Lemma 2.6, we thus have that $D^{2} \stackrel{C_{4}}{\sim} C \cdot G^{-1}$, where $G^{-1}$ denotes the inverse of the central element $G$ in $\mathcal{S L}(2)$. The result follows.

Remark 3.6 The techniques used in Section 3.1 to prove that $\mathcal{S L}(2) / C_{k}$ is abelian for $k \leq 6$ can also be applied to the case $k=7$ to some extent. More precisely, by fixing a generating set $\mathcal{H}_{6}$ for $\mathcal{S L}_{6}(2) / C_{7}$, one can try to show directly that lifts to $\mathcal{S L}(2) / C_{7}$ of any two elements of $\bigcup_{i=1}^{6} \mathcal{H}_{i}$ commute. We can actually apply our methods, involving somewhat advanced calculus of claspers, to prove that this is indeed the case, except for one computation that remains open. Namely, the question whether $H \cdot Y$ is $C_{7}$-equivalent to $Y \cdot H$ remains open so far, and is the only missing case to establish the commutativity of $\mathcal{S L}(2) / C_{7}$ (without the 2-torsion assumption). 


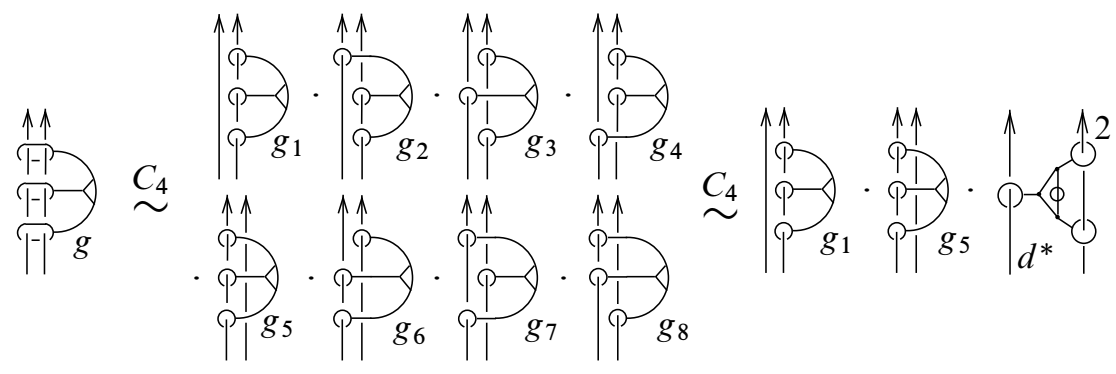

Figure 3.10

\section{The group of $C_{k}$-concordance classes of string links}

In this section, we prove Theorem 1.8 and Proposition 1.1. We start with a brief review on the $C_{k}$-concordance.

\section{1 $C_{k}$-concordance}

Let $k$ and $n$ be positive integers. Two $n$-string links $L, L^{\prime}$ are $C_{k}$-concordant if there is a sequence $L=L_{0}, L_{1}, \ldots, L_{m}=L^{\prime}$ of $n$-string links such that for each $i \geq 1$, either

$$
L_{i} \stackrel{C_{k+1}}{\sim} L_{i+1}
$$

or $L_{i}$ is concordant to $L_{i+1}$. We denote the $C_{k}$-concordance relation by $\stackrel{C_{k}+c}{\sim}$.

A string link is $\left(C_{k}+c\right)$-trivial if it is $C_{k}$-concordant to the trivial string link.

It is well known that Milnor invariants are concordance invariants; see Casson [4]. So by [13, Theorem 7.1], Milnor invariants of length less than or equal to $k$ are $C_{k}-$ concordance invariants. Habegger and Masbaum showed that all rational finite-type concordance invariants of string links are given by Milnor invariants via the Kontsevich integral [12].

It is known that surgery along graph claspers with loops (ie graph claspers that are not tree claspers) implies concordance.

Lemma 4.1 (Conant and Teichner [7], Garoufalidis and Levine [9]) For any graph clasper with loop $g$ for $\mathbf{1}$, the string link $\mathbf{1}_{g}$ is concordant to $\mathbf{1}$.

By combining this lemma and the STU relation, we have the following. 
Lemma 4.2 Let $g_{T}$ and $g_{U}$ be two $C_{k}$-trees for 1 which differ only in a small ball as depicted in Figure 2.4. Then

$$
\mathbf{1}_{g_{T}} \stackrel{C_{k+1}+c}{\sim} \mathbf{1}_{g_{U}}
$$

Note also that the algebraic argument used to prove Lemma 2.9 also apply in this context, so that there is a ' $C_{k}$-concordance version' of Lemma 2.9 (see Lemma 4.7 below).

\subsection{The ordered index}

In order to study $C_{k}$-concordance for string links, we use the notion of ordered index of a linear $C_{k}$-tree.

Let $t$ be a simple tree clasper for a string link $L$. We call a leaf of $t$ an $i$-leaf if it intersects the $i^{\text {th }}$ component of $L$. The index of $t$ is the collection of all integers $i$ such that $t$ contains an $i$-leaf, counted with multiplicities. For example, a simple $C_{3}$-tree of index $\left\{2,3^{(2)}, 5\right\}$ for $L$ intersects component 3 twice and components 2 and 5 once (and is disjoint from all other components of $L$ ).

For $k \geq 3$, a $C_{k}$-tree $G$ having the shape of the tree clasper in Figure 2.1 is called a linear $C_{k}$-tree. (Note in particular that a linear tree clasper is always assumed to be simple.) The left-most and right-most leaves of $G$ in Figure 2.1 are called the ends of $G$. As a convention, any simple $C_{k}$-tree is linear for $k \leq 2$; the ends of a linear $C_{1}$-tree (resp. $C_{2}$-tree) are its two leaves (resp. a choice of any two leaves).

Let $t$ be a linear $C_{k}$-tree with ends $f_{0}, f_{k}$. Since $t$ is a disk, we can travel from $f_{0}$ to $f_{k}$ along $\partial t$ so that we meet all other leaves $f_{1}, \ldots, f_{k-1}$ in this order. If $f_{s}$ is an $i_{s}$-leaf $(s=0, \ldots, k)$, we can consider two vectors $\left(i_{0}, \ldots, i_{k}\right)$ and $\left(i_{k}, \ldots, i_{0}\right)$ and may assume that $\left(i_{0}, \ldots, i_{k}\right) \leq\left(i_{k}, \ldots, i_{0}\right)$, where ' $\leq$ ' is the lexicographic order in $\mathbb{Z}^{k+1}$. We call $\left(i_{0}, \ldots, i_{k}\right)$ the ordered index of $t$ and denote it by o-index $(t)$. In the following, we will simply denote by $\left(i_{0} \ldots i_{k}\right)$ an ordered index $\left(i_{0}, \ldots, i_{k}\right)$.

By combining Lemma 4.1 and [17, Lemma 5.1], we have the following lemma.

Lemma 4.3 (1) Let $t$ and $t^{\prime}$ be two linear $C_{k}$-trees for 1 with same ordered index. Then either

$$
\mathbf{1}_{t} \stackrel{C_{k+1}+c}{\sim} \mathbf{1}_{t^{\prime}} \text { or } \mathbf{1}_{t} \cdot \mathbf{1}_{t^{\prime}} \stackrel{C_{k+1}+c}{\sim} \mathbf{1} .
$$

(2) Let $t$ be a linear $C_{k}$-tree $(k \geq 3)$ for 1 with o-index $(t)=\left(i_{0} \ldots i_{k}\right)$. If $i_{0}=i_{1}$ or $i_{k-1}=i_{k}$, then

$$
\mathbf{1}_{t} \stackrel{C_{k+1}+c}{\sim} \mathbf{1}
$$


For each sequence $I=\left(i_{0} \ldots i_{k}\right)$ of integers in $\{1,2\}$, let $T(I)$ denote the choice of a 2 -string link obtained from $\mathbf{1}_{2}$ by surgery along a linear $C_{k}$-tree with o-index $I$. In particular, the $2-$ string links $T(12), T(121), T(1221), T(12221)$ and $T(21112)$ are chosen to be the string links $I, Y, H, S_{\text {id }}^{1}$ and $S_{\text {id }}^{2}$ introduced in Section 3.1, respectively. We note that by Lemma 4.3(1), there are essentially two choices in $\mathcal{S L}(2) /\left(C_{k+1}+c\right)$ for each sequence $I$.

\subsection{Proofs of Proposition 1.1 and Theorem 1.8(2)}

We first show why $\mathcal{S L}(n) /\left(C_{3}+c\right)$ is not abelian for any $n \geq 3$.

Since the $C_{k}$-concordance implies the $C_{m}$-concordance if $k>m$, this will imply statement (2) of Theorem 1.8, and since the $C_{k}$-equivalence implies the $C_{k}$-concordance, we will also deduce Proposition 1.1.

Let $\sigma_{1}$ and $\sigma_{2}$ be the Artin generators for the 3-braid group. Then $\sigma_{1}^{2}, \sigma_{1}^{-2}, \sigma_{2}^{2}$ and $\sigma_{2}^{-2}$ are 3 -string links. Since the closure of $\sigma_{1}^{2} \sigma_{2}^{2} \sigma_{1}^{-2} \sigma_{2}^{-2}$ is a copy of the Borromean rings, $\sigma_{1}^{2} \sigma_{2}^{2} \sigma_{1}^{-2} \sigma_{2}^{-2}$ has nontrivial Milnor invariant $\mu(123)$ of length 3, which is a $C_{3}$-concordance invariant. Hence $\sigma_{1}^{2} \sigma_{2}^{2} \sigma_{1}^{-2} \sigma_{2}^{-2}$ is not $C_{3}$-concordant to $\mathbf{1}_{3}$. This implies that $\mathcal{S L}(3) /\left(C_{3}+c\right)$ is not commutative: suppose that $\sigma_{1}^{2}$ and $\sigma_{2}^{2}$ commute in $\mathcal{S L}(3) /\left(C_{3}+c\right)$, then $\sigma_{1}^{2} \sigma_{2}^{2} \sigma_{1}^{-2} \sigma_{2}^{-2}$ is $C_{3}$-concordant to $\sigma_{2}^{2} \sigma_{1}^{2} \sigma_{1}^{-2} \sigma_{2}^{-2}=\mathbf{1}_{3}$, which leads to a contradiction.

\subsection{Abelian cases: $k \leq 8$}

We now show that $\mathcal{S L}(2) /\left(C_{k}+c\right)$ is abelian for $k \leq 8$.

Since the $C_{k}$-concordance implies the $C_{m}$-concordance for $k>m$, it is sufficient to show that $\mathcal{S L}(2) /\left(C_{8}+c\right)$ is abelian.

We use the same strategy as in Section 3.1, where we showed that $\mathcal{S L}(2) / C_{6}$ is abelian. More precisely, we first chose generating sets for the successive quotients $\mathcal{S L}_{i}^{c}(2) /\left(C_{i+1}+c\right)(i \leq 7)$, where $\mathcal{S L}_{i}^{c}(2)$ is the set of $\left(C_{i}+c\right)$-trivial 2-string links, to obtain a set of generators for $\mathcal{S L}(2) /\left(C_{8}+c\right)$, then we show that any two of these generators commute. In this discussion, we may again ignore local generators, since they are central in $\mathcal{S L}(2)$.

Let

$$
\begin{gathered}
\mathcal{H}_{1}^{c}=\{T(12)\}, \quad \mathcal{H}_{2}^{c}=\{T(121)\}, \quad \mathcal{H}_{3}^{c}=\{T(1221)\}, \\
\mathcal{H}_{4}^{c}=\{T(12221), T(21112)\}, \quad \mathcal{H}_{5}^{c}=\{T(122221), T(211112), T(121221)\} .
\end{gathered}
$$


In [17], the authors show that $\mathcal{H}_{i}^{c}$ is a generating set for $\mathcal{S L}_{i}^{c}(2) /\left(C_{i+1}+c\right), i \leq 5.4$ Let us now pick a generating set for $\mathcal{S L}_{6}^{c}(2) /\left(C_{7}+c\right)$. By Lemma 4.3(2), a string link obtained from $\mathbf{1}_{2}$ by surgery along a linear $C_{6}$-tree with index $\left\{1,2^{(6)}\right\}$ or $\left\{1^{(6)}, 2\right\}$ is $C_{7}$-concordant to $\mathbf{1}_{2}$. So it is enough to consider $C_{6}$-trees with index $\left\{1^{(2)}, 2^{(5)}\right\}$, $\left\{1^{(3)}, 2^{(4)}\right\},\left\{1^{(4)}, 2^{(3)}\right\}$ or $\left\{1^{(5)}, 2^{(2)}\right\}$. Furthermore, the IHX relation implies that it is sufficient to consider linear $C_{6}$-trees.

By the IHX relation, the ends of a linear $C_{6}$-trees with index $\left\{1^{(2)}, 2^{(5)}\right\}$ can be chosen to be the two 1-leaves, so that the only possible o-index is (1222221). Similarly, we may assume that the ends of a linear $C_{6}$-trees with index $\left\{1^{(3)}, 2^{(4)}\right\}$ are both 2-leaves. Then by Lemma 4.3(2), the o-index should be of the form (21ijk12) for some $i, j, k \in\{1,2\}$. Since the index is $\left\{1^{(3)}, 2^{(4)}\right\}$, we have three possibilities, namely $i j k=212,122$, or 221 . By definition, the only two possible o-indices are then (2112212) and (2121212). Summarizing, we may assume that

(1) all linear $C_{6}$-trees with index $\left\{1^{(2)}, 2^{(5)}\right\}$ have o-index (1222221),

(2) all linear $C_{6}$-trees with index $\left\{1^{(3)}, 2^{(4)}\right\}$ have o-index (2121212) or (2112212),

(3) all linear $C_{6}$-trees with index $\left\{1^{(4)}, 2^{(3)}\right\}$ have o-index (1212121) or (1211221),

(4) all linear $C_{6}$-trees with index $\left\{1^{(5)}, 2^{(2)}\right\}$ have o-index (2111112).

(The last two cases are deduced from the first two by exchanging 1 and 2.)

The following lemma is useful to further reduce the number of generators.

Lemma 4.4 For an integer $k \geq 5$, let $t$ and $t^{\prime}$ be two linear $C_{k}$-trees for $\mathbf{1}_{2}$ whose respective $o$-indices are either of the form (ijjiiI) and (ijijiI), where $I$ is a sequence of $k-4$ integers in $\{1,2\}$ and where $\{i, j\}=\{1,2\}$. Then either

$$
\left(\mathbf{1}_{2}\right)_{t} \stackrel{C_{k+1}+c}{\sim}\left(\mathbf{1}_{2}\right)_{t^{\prime}} \text { or }\left(\mathbf{1}_{2}\right)_{t} \cdot\left(\mathbf{1}_{2}\right)_{t^{\prime}} \stackrel{C_{k+1}+c}{\sim} \mathbf{1}_{2} \text {. }
$$

Proof Suppose that o-index $(t)$ is of the form (ijji i I). By Lemmas 4.2 and 2.6, we can assume without loss of generality that there a 3-ball which intersects $\mathbf{1}_{2} \cup t$ as shown on the left-hand side of Figure 4.1. Using Lemma 2.6 again, we have that $\left(\mathbf{1}_{2}\right)_{t}$ is $C_{k+1}$-equivalent to $\left(\mathbf{1}_{2}\right)_{t^{\prime}}$, where $t^{\prime}$ is shown in the figure.

By the IHX relation, $\left(\mathbf{1}_{2}\right)_{t^{\prime}}$ is $C_{k+1}$-equivalent to $L \cdot S$, where $L$ and $S$ are string links as illustrated in Figure 4.1. Notice that, by the AS relation, $L$ is $C_{k+1}$-equivalent to a string link obtained from $\mathbf{1}_{2}$ by surgery along a $C_{k}$-trees with o-index ( $\left.i j i j i I\right)$. So, proving that $S$ is $\left(C_{k+1}+c\right)$-trivial would imply Lemma 4.4.

${ }^{4}$ In [17], $T(121212)$ is chosen instead of $T(121221)$, but these two string links are $C_{6}$-equivalent by the AS relation. 

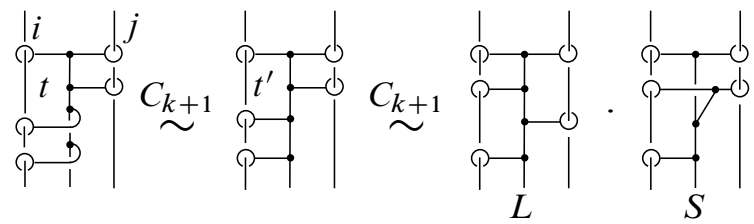

Figure 4.1

We now show that $S$ is indeed $\left(C_{k+1}+c\right)$-trivial. The proof is given in Figure 4.2 as follows. By Lemma 2.6 and the IHX relation, we have

$$
S \stackrel{C_{k+1}}{\sim} U \cdot V
$$

where $U$ and $V$ are as shown, and by Lemmas 2.3 and 4.2, $V$ is $C_{k+1}$-concordant to the string link $W$ represented on the right-hand side.
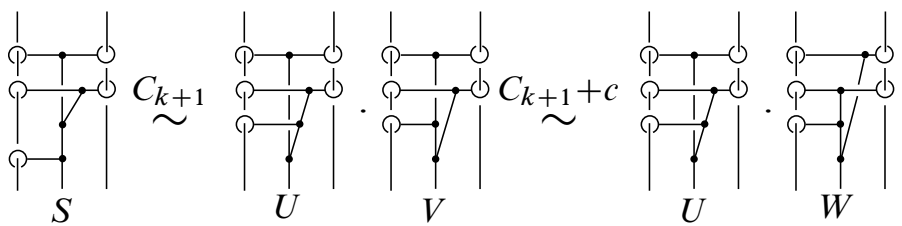

Figure 4.2

It then follows from the AS relation that $U \cdot W^{C_{k+1}} \mathbf{1}_{2}$, which concludes the proof.

Remark 4.5 The same result holds if $t$ and $t^{\prime}$ have respective o-indices ( $I i j j \mathrm{ji}$ ) and $($ I ijiji).

Using this, we can assume all linear $C_{6}$-trees with index $\left\{1^{(3)}, 2^{(4)}\right\}$ and $\left\{1^{(4)}, 2^{(3)}\right\}$ have o-index (2121212) and (1212121), respectively. It follows that

$$
\mathcal{H}_{6}^{c}=\{T(1222221), T(1212121), T(2121212), T(2111112)\}
$$

is a generating set for $\mathcal{S L}_{6}^{c}(2) /\left(C_{7}+c\right)$.

Similarly, we have the following generating set for $\mathcal{S L}_{7}^{c}(2) /\left(C_{8}+c\right)$ :

$\mathcal{H}_{7}^{c}=\{T(1222221), T(21111112), T(12111221), T(21122212), T(12211221)$,

$T(12112221)\}$

In the following, we call generator of degree $k(k \leq 7)$ any element $T(I)$ of the generating set $\mathcal{H}_{k}^{c}$. 
In order to prove that $\mathcal{S L}(2) /\left(C_{8}+c\right)$ is abelian, it suffices to show that any two generators of degree less than or equal to 7 do commute in $\mathcal{S L}(2) /\left(C_{8}+c\right)$.

By Lemma 3.1, $T(12)$ is central in $\mathcal{S L}(2)$. Moreover by Remark 2.4, two generators of degrees $k$ and $l$ commute in $\mathcal{S L}(2) /\left(C_{8}+c\right)$ if $k+l \geq 8$. Hence it is enough to check the commutativity of generators of degrees $k$ and $l$ for $(k, l)=$ $(3,4),(2,5),(2,4),(2,3)$. (The cases $(3,3)$ and $(2,2)$ are vacuous, since there is only one generator in degree 3 and 2.)

In order to prove the commutativity in the case $(3,4)$, we need the following.

Lemma 4.6 Let $t$ be a linear $C_{k}$-tree $(k \geq 1)$ for $\mathbf{1}_{n}$ with $k$ odd. Then $\left(\mathbf{1}_{n}\right)_{t}$ is $C_{k+1}$-concordant to its image under orientation-reversal of all strings.

A proof is easily obtained by combining Lemma 4.1 and arguments similar to those in the proof of [17, Lemma 5.1 (3)]. Here, let us only illustrate the general idea on an example. Consider the linear $C_{7}$-tree $t$ for $\mathbf{1}_{2}$ illustrated on the left-hand side of Figure 4.3. Let also $t^{\prime}$ be the linear $C_{7}$-tree for $\mathbf{1}_{2}$ illustrated on the right-hand side of the figure. (notice that both $t$ and $t^{\prime}$ have o-index (12112221).) On one hand, the two string links obtained by surgery along $t$ and $t^{\prime}$ are obtained from one another by reversing the orientation of all strings. On the other hand, by Lemma 2.6 we have that $\left(\mathbf{1}_{2}\right)_{t}$ is $C_{8}$-equivalent to $\left(\mathbf{1}_{2}\right)_{c}$, where $c$ is the $C_{7}$-tree shown in the figure. Let $\tilde{c}$ be obtained by a 180-degree rotation of $c$ around the axis $a$ fixing the leaves; see Figure 4.3. By sliding the leaves of $\tilde{c}$ repeatedly, we can deform it into the $C_{7}$-tree $c^{\prime}$
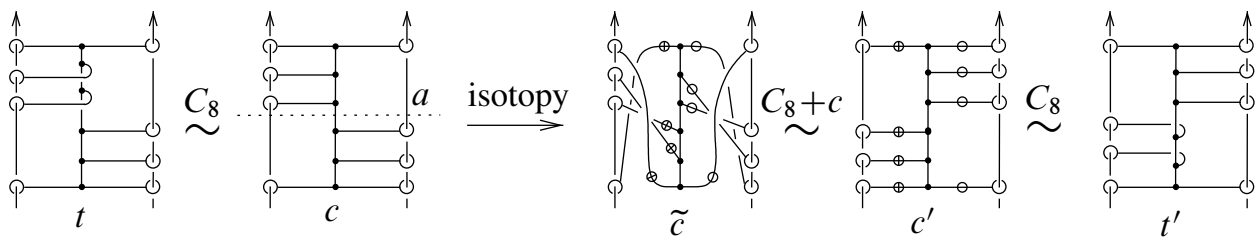

Figure 4.3: The two string links obtained by surgery along $t$ and $t^{\prime}$ are $C_{8}$-concordant.

shown in Figure 4.3, which only differs from $t^{\prime}$ by an even number of half-twists on its edges. By Lemmas 4.2 and 2.6, we obtain that $\left(\mathbf{1}_{2}\right)_{t}$ and $\left(\mathbf{1}_{2}\right)_{t^{\prime}}$ are $C_{8}$-concordant. (In the general case, the fact that the degree is odd ensures that there is an even number of half-twists.)

We can now prove the desired commutativity property in the case $(k, l)=(3,4)$.

Case $(3,4)$ We first show that $T(1221)$ and $T(12221)$ commute in $\mathcal{S L}(2) /\left(C_{8}+c\right)$. Since both string links are symmetric, we note that $T(12221) \cdot T(1221)$ is obtained 
from $T(1221) \cdot T(12221)$ by orientation-reversal of both strings. By Lemma 2.2, $T(1221) \cdot T(12221)$ is $C_{8}$-equivalent to $\left(\mathbf{1}_{2}\right)_{t \cup s} \cdot L$, where $t$ and $s$ are tree claspers for $\mathbf{1}_{2}$ as illustrated in Figure 4.4, and where $L$ is a string link obtained from $\mathbf{1}_{2}$ by surgery along some $C_{7}$-trees. Since $L$ is $C_{8}$-concordant to a product $L^{\prime}$ of generators

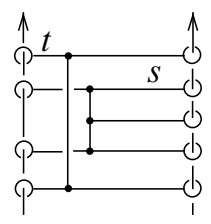

Figure 4.4

of degree 7 , we have that $T(1221) \cdot T(12221)$ is $C_{8}$-concordant to $\left(\mathbf{1}_{2}\right)_{t \cup s} \cdot L^{\prime}$. Hence $T(12221) \cdot T(1221)$ is $C_{8}$-concordant to the 2 -string link $\left(\mathbf{1}_{2}\right)_{t \cup s} \cdot L^{\prime}$ with orientation reversed. On the other hand, by Lemma $2.3,\left(\mathbf{1}_{2}\right)_{t \cup s}$ is $C_{8}$-equivalent to its image under orientation-reversal, and by Lemma 4.6, each generator of degree 7 is $C_{8}-$ concordant to its image under orientation-reversal. It follows that $T(1221) \cdot T(12221)$ and $T(12221) \cdot T(1221)$ are $C_{8}$-concordant.

The fact that $T(1221)$ and $T(21112)$ commute in $\mathcal{S L}(2) /\left(C_{8}+c\right)$ is shown completely similarly.

Before we deal with the remaining cases, we make an observation. Since the group $\mathcal{S L}_{7}^{c}(2) /\left(C_{8}+c\right)$ is generated by the 6 elements of $\mathcal{H}_{7}^{c}$, and since there are 6 independent Milnor invariants of length 8 (see Cochran [5, Appendix B]), we have that $\mathcal{S L}_{7}^{c}(2) /\left(C_{8}+c\right)$ is a free abelian group with rank 6 , and in particular, has no 2-torsion. Moreover, by Claim 3.5, we have that $T(121)^{2}\left(=Y^{2}\right)$ is $C_{3}$-equivalent to a central element in $\mathcal{S L}(2)$. So we get the following as an application of the ' $C_{k}$-concordance version' of Lemma 2.9 (see also Remark 2.11).

Lemma 4.7 Suppose that the string link $T(121)$ commutes with any $\left(C_{l}+c\right)$-trivial string link in $\mathcal{S L}(n) /\left(C_{7}+c\right)$, for some integers $l(l<8)$. Suppose moreover that $\left(C_{3}+c\right)$-trivial and $\left(C_{l}+c\right)$-trivial string links commute in $\mathcal{S L}(n) /\left(C_{8}+c\right)$. Then $T(121)$ commutes with any $\left(C_{l}+c\right)$-trivial string link in $\mathcal{S L}(n) /\left(C_{8}+c\right)$.

We can now prove the desired commutativity property in the remaining cases $(k, l)=$ $(2,5),(2,4),(2,3)$, namely that $T(121)$ commutes with generators of degree 5,4 and 3 in $\mathcal{S L}(2) /\left(C_{8}+c\right)$.

Case $(2,5)$ By Remark 2.4, $T(121)$ commutes with any $C_{5}$-trivial string link in $\mathcal{S L}(2) / C_{7}$. Also, any $C_{3}$-trivial string link commutes with any $C_{5}$-trivial string link 
in $\mathcal{S L}(2) / C_{8}$. So by applying Lemma 4.7 for $l=5$, we have that $T(121)$ commutes with any $\left(C_{5}+c\right)$-trivial string link in $\mathcal{S L}(2) /\left(C_{8}+c\right)$.

Case $(2,4)$ We already showed that any $\left(C_{3}+c\right)$-trivial string link commutes with any $\left(C_{4}+c\right)$-trivial string link in $\mathcal{S L}(2) /\left(C_{8}+c\right)$ (Case $\left.(3,4)\right)$. By an argument similar to that of Case $(3,4)$ above, one can easily show that $T(121)$ commutes with any $C_{4}$-trivial string link in $\mathcal{S L}(2) /\left(C_{7}+c\right)$. By applying Lemma 4.7 for $l=4$, we thus have that $T(121)$ commutes with any $\left(C_{4}+c\right)$-trivial string link in $\mathcal{S L}(2) /\left(C_{8}+c\right)$.

Case $(2,3)$ We only have to show that $T(121)$ and $T(1221)$ (that is, $Y$ and $H$ ) commute in $\mathcal{S L}(2) /\left(C_{7}+c\right)$, since this and Lemma 4.7 for $l=3$ implies the desired commutativity property. To do so, recall that we showed in the proof of Proposition 3.3 that $Y \cdot H$ is $C_{6}$-equivalent $A \cdot C \cdot D \cdot E$ (see Figures 3.4 and 3.5), which implies that $Y \cdot H$ is $C_{7}$-concordant to $A \cdot C \cdot D \cdot E \cdot L$, where $L$ is a product of string links, each obtained by surgery along a degree 6 generator. Since all such generators are symmetric, as well as $Y, H, C, D$ and $E$, we have by Remark 2.4 that $H \cdot Y=\overline{Y \cdot H}$ is $C_{7}$-concordant to $\bar{A} \cdot C \cdot D \cdot E \cdot L$. Hence it suffices to show that $A$ and $\bar{A}$ are $C_{7}$-concordant. To see this, notice that the tree claspers defining $A$ and $\bar{A}$ only differ by a crossing change between edges, so that by Lemma 2.3, $A$ is $C_{7}$-equivalent to a product $\bar{A} \cdot\left(\mathbf{1}_{2}\right)_{T}$, where $T$ is a $C_{6}$-tree that intersects a ball as shown on the left-hand side of Figure 2.5. The result then follows by combining Lemmas 2.8 and 4.1.

This concludes the proof that $\mathcal{S L}(2) /\left(C_{k}+c\right)$ is abelian for all $k \leq 8$.

\subsection{Nonabelian case}

Let $L_{1}, L_{1}^{\prime}, L_{2}$ and $L_{2}^{\prime}$ be 2-string links as illustrated in Figure 4.5. The following
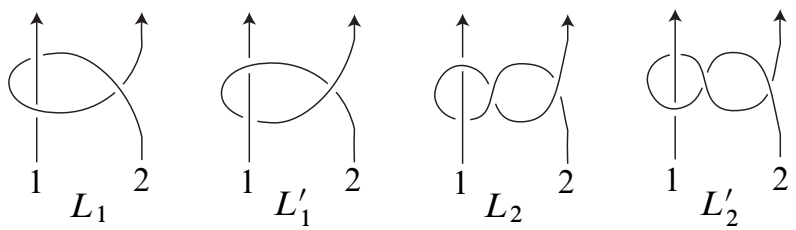

Figure 4.5

implies that $\mathcal{S L}(2) /\left(C_{k}+c\right)$ is not abelian for all $k \geq 9$.

Proposition 4.8 The string links $L_{1}$ and $L_{2}$ do not commute in $\mathcal{S L}(2) /\left(C_{9}+c\right)$. 
Proof The proof relies on a direct computation, using a program by Takabatake, Kuboyama and Sakamoto [24] ${ }^{5}$ based on the algorithm given by Milnor in [18] to compute $\mu$-invariants. We have that the Magnus expansion of the $1^{\text {st }}$ longitude of $L_{1} \cdot L_{2} \cdot L_{1}^{\prime} \cdot L_{2}^{\prime}$ is

$1-X X X Y X Y Y Y+X X X Y Y Y X Y+5 X X Y X X Y Y Y$

$-6 X X Y X Y X Y Y-X X Y X Y Y Y X+X X Y X Y Y Y Y+6 X X Y Y X Y X Y$

$-5 X X Y Y X Y Y Y-5 X X Y Y Y X X Y+X X Y Y Y X Y X+5 X X Y Y Y X Y Y$

$-X X Y Y Y Y X Y-5 X Y X X X Y Y Y+9 X Y X X Y X Y Y-9 X Y X X Y Y X Y$

$+5 X Y X X Y Y Y X-6 X Y X Y X Y Y X+6 X Y X Y X Y Y Y+9 X Y X Y Y X X Y$

- $9 X Y X Y Y X Y Y-X Y X Y Y Y X X+X Y X Y Y Y Y X-9 X Y Y X Y X X Y$

+6XYYXYXYX+9XYYXYYXY-5XYYXYYYX+5XYYYXXXY

$-5 X Y Y Y X X Y X+X Y Y Y X Y X X-6 X Y Y Y X Y X Y+5 X Y Y Y X Y Y X$

- XYYYYXYX+YXXXXYYY+6YXXXYYXY-5YXXXYYYX

$-Y X X X Y Y Y Y-9 Y X X Y X X Y Y+9 Y X X Y X Y Y X-9 Y X X Y Y X Y X$

$+9 Y X X Y Y X Y Y+5 Y X X Y Y Y X X-6 Y X X Y Y Y X Y+6 Y X Y X X X Y Y$

$-6 Y X Y X X Y Y Y-6 Y X Y X Y Y X X+6 Y X Y X Y Y Y X-6 Y X Y Y X X X Y$

+9YXYYXXYX-9YXYYXYYX-YXYYYXXX+6YXYYYXXY

$+Y X Y Y Y Y X X-6 Y Y X X X Y X Y+5 Y Y X X X Y Y Y+9 Y Y X X Y X X Y$

$-9 Y Y X X Y X Y Y-9 Y Y X Y X X Y X+9 Y Y X Y X X Y Y+6 Y Y X Y X Y X X$

$-9 Y Y X Y Y X X Y+9 Y Y X Y Y X Y X-5 Y Y X Y Y Y X X-Y Y Y X X X X Y$

$+5 Y Y Y X X X Y X-5 Y Y Y X X X Y Y-5 Y Y Y X X Y X X+6 Y Y Y X X Y X Y$

$+Y Y Y X Y X X X-6 Y Y Y X Y X Y X+5 Y Y Y X Y Y X X+Y Y Y Y X X X Y$

$-Y Y Y Y X Y X X+$ (higher-degree terms),

where the Magnus expansion is defined here by sending the first meridian to $1+X$ and the second meridian to $1+Y$. Hence some Milnor invariants of length 9 do not vanish on the string link $L_{1} \cdot L_{2} \cdot L_{1}^{\prime} \cdot L_{2}^{\prime}$. This implies that $L_{1} \cdot L_{2} \cdot L_{1}^{\prime} \cdot L_{2}^{\prime}$ is not $C_{9}$-concordant to $\mathbf{1}_{2}$. Now, if $L_{1}$ commutes with $L_{2}$ in $\mathcal{S L}(2) /\left(C_{9}+c\right)$, then

5 The first version, based on Milnor's algorithm, was written by Tetsuji Kuboyama using the programming language Ruby. The current version [24] is a significantly improved version of it, due to Yoshimasa Takabatake, Tetsuji Kuboyama and Hiroshi Sakamoto, which uses C++. 
$L_{1} \cdot L_{2} \cdot L_{1}^{\prime} \cdot L_{2}^{\prime}$ is $C_{9}$-concordant to $L_{2} \cdot L_{1} \cdot L_{1}^{\prime} \cdot L_{2}^{\prime}$, which is concordant to $\mathbf{1}_{2}$. This is a contradiction.

\section{Appendix: Proofs of Corollaries 1.6 and 1.9}

In this short appendix, we briefly outline the proofs of Corollaries 1.6 and 1.9. That is, we show how the abelian group structure on the set of $C_{k}$-equivalence classes (resp. $C_{k}$-concordance classes) of 2-string links implies the Goussarov-Habiro Conjecture at the corresponding degree. As explained in the introduction, this is merely an adaptation of Habiro's argument for proving Theorem 1.5, so we do not reproduce here all the details of this proof, but give precise references to the technical results from Habiro's paper that are used in this arguments, and emphasize the role of the commutativity of $\mathcal{S L}(n) / C_{k+1}$.

For simplicity, we only give the arguments for Corollary 1.6. They easily adapt to the case of $C_{k}$-concordance and finite-type concordance invariants to obtain Corollary 1.9. Actually, Habiro's proof of Theorem 1.5 can be adapted to show the following.

Proposition A.1 Suppose that the group of $C_{k+1}$-equivalence classes of $n$-string links is abelian. Then two $n$-string links cannot be distinguished by any finite-type invariant of order less than or equal to $k$ if and only if they are $C_{k+1}$-equivalent.

Proof The fact that two $C_{k+1}$-equivalent string links share all finite-type invariants of degree up to $k$ is well known [13, Corollary 6.8], so we only need to show the converse implication.

Suppose that the group $\mathcal{S L}(n) / C_{k+1}$ is abelian. Following Habiro's strategy for proving Theorem 1.5 [13, Theorem 6.18], we consider the homomorphism of abelian groups (from an additive to a multiplicative abelian group)

$$
\varphi_{k}: \mathbb{Z} \mathcal{S L}(n) \longrightarrow \mathcal{S L}(n) / C_{k+1}
$$

which maps an $n$-string link $L$ to its $C_{k+1}$-equivalence class $[L]_{k+1}$. The key point is that the existence (ie the well-definedness) of this map relies on the fact that $\mathcal{S} \mathcal{L}(n) / C_{k+1}$ is abelian. Notice that $\varphi_{k}$ is indeed additive, since for two $n$-string links $L$ and $L^{\prime}$, we have

$$
\varphi_{k}\left(L \cdot L^{\prime}-L-L^{\prime}\right)=\left[L \cdot L^{\prime}\right]_{k+1} \cdot[L]_{k+1}^{-1} \cdot\left[L^{\prime}\right]_{k+1}^{-1}=[\mathbf{1}]_{k+1}=\varphi_{k}(\mathbf{1}) .
$$

Now, we have that $\varphi_{k}$ is a finite-type invariant of degree $k$. This is proved by Habiro in the (string) knot case in [13, Proposition 6.16]. His argument relies on a deep 
result on the structure of the Goussarov-Vassiliev filtration [13, Proposition 6.10], which involves advanced clasper theory. But Habiro actually established the latter result not only for (string) knots, but also for string links (and more generally for surface string links). So we can freely use [13, Proposition 6.10] to show that $\varphi_{k}$ is a finite-type invariant of degree $k$. The proof of Proposition A. 1 is then completed by simply following [13, Theorem 6.18] as follows. If two $n$-string links $L$ and $L^{\prime}$ cannot be distinguished by any finite-type invariant of order less than or equal to $k$, then $\varphi_{k}(L)=\varphi_{k}\left(L^{\prime}\right)$. This implies that $[L]_{k+1}=\left[L^{\prime}\right]_{k+1}$, that is, $L$ and $L^{\prime}$ are $C_{k+1}$-equivalent.

\section{References}

[1] D Bar-Natan, On the Vassiliev knot invariants, Topology 34 (1995) 423-472 MR1318886

[2] D Bar-Natan, Vassiliev and quantum invariants of braids, from: "The interface of knots and physics", (L H Kauffman, editor), Proc. Sympos. Appl. Math. 51, Amer. Math. Soc. (1996) 129-144 MR1372767

[3] J E P P de Campos, Boundary theta curves in $S^{3}$, Tokyo J. Math. 28 (2005) 283-288 MR2149637

[4] A J Casson, Link cobordism and Milnor's invariant, Bull. London Math. Soc. 7 (1975) 39-40 MR0362286

[5] T D Cochran, Derivatives of links: Milnor's concordance invariants and Massey's products, Mem. Amer. Math. Soc. 427, Amer. Math. Soc. (1990) MR1042041

[6] J Conant, R Schneiderman, P Teichner, Whitney tower concordance of classical links, Geom. Topol. 16 (2012) 1419-1479 MR2967057

[7] J Conant, P Teichner, Grope cobordism of classical knots, Topology 43 (2004) 119156 MR2030589

[8] S V Duzhin, M V Karev, Determination of the orientation of string links using finitetype invariants, Funktsional. Anal. i Prilozhen. 41 (2007) 48-59 MR2381954

[9] S Garoufalidis, J Levine, Concordance and 1-loop clovers, Algebr. Geom. Topol. 1 (2001) 687-697 MR1875612

[10] M N Gusarov, Variations of knotted graphs: The geometric technique of n-equivalence, Algebra i Analiz 12 (2000) 79-125 MR1793618

[11] N Habegger, X-S Lin, On link concordance and Milnor's $\bar{\mu}$ invariants, Bull. London Math. Soc. 30 (1998) 419-428 MR1620841

[12] N Habegger, G Masbaum, The Kontsevich integral and Milnor's invariants, Topology 39 (2000) 1253-1289 MR1783857 
[13] K Habiro, Claspers and finite type invariants of links, Geom. Topol. 4 (2000) 1-83 MR1735632

[14] J-Y Le Dimet, Cobordisme d'enlacements de disques, Mém. Soc. Math. France 32, Soc. Math. France, Paris (1988) MR971415

[15] G Massuyeau, Finite-type invariants of 3-manifolds and the dimension subgroup problem, J. Lond. Math. Soc. 75 (2007) 791-811 MR2352736

[16] J-B Meilhan, On Vassiliev invariants of order two for string links, J. Knot Theory Ramifications 14 (2005) 665-687 MR2162120

[17] J-B Meilhan, A Yasuhara, Characterization of finite type string link invariants of degree < 5, Math. Proc. Cambridge Philos. Soc. 148 (2010) 439-472 MR2609302

[18] J Milnor, Isotopy of links: Algebraic geometry and topology, from: "A symposium in honor of S Lefschetz”, Princeton Univ. Press (1957) 280-306 MR0092150

[19] K Miyazaki, The theta-curve cobordism group is not abelian, Tokyo J. Math. 17 (1994) 165-169 MR1279576

[20] H Murakami, Y Nakanishi, On a certain move generating link-homology, Math. Ann. 284 (1989) 75-89 MR995383

[21] K Y Ng, Groups of ribbon knots, Topology 37 (1998) 441-458 MR1489214

[22] T Ohtsuki, Quantum invariants: A study of knots, 3-manifolds, and their sets, Series on Knots and Everything 29, World Scientific, River Edge, NJ (2002) MR1881401

[23] T Stanford, Braid commutators and Vassiliev invariants, Pacific J. Math. 174 (1996) 269-276 MR1398378

[24] Y Takabatake, T Kuboyama, H Sakamoto, stringcmp: Faster calculation for Milnor invariant Available at https://code.google.com/p/stringcmp/

[25] A Yasuhara, $C_{k}$-moves on spatial theta-curves and Vassiliev invariants, Topology Appl. 128 (2003) 309-324 MR1957422

Institut Fourier, Université Grenoble 1

100 rue des Maths, BP 74, 38402 Saint Martin d'Hères, France

Department of Mathematics, Tokyo Gakugei University

4-1-1 Nukuikita-Machi, Koganei-shi, Tokyo 184-8501, Japan

jean-baptiste.meilhan@ujf-grenoble.fr, yasuhara@u-gakugei.ac.jp

http://www-fourier.ujf-grenoble.fr/ meilhan/,

http://www.u-gakugei.ac.jp/ yasuhara/

Received: 7 May $2013 \quad$ Revised: 31 October 2013 